$\begin{array}{cl}\text { PRAMANA } & \text { (C) Indian Academy of Sciences } \\ \begin{array}{c}\text { journal of } \\ \text { physics }\end{array} & \begin{array}{l}\text { Vol. 69, No. } 5 \\ \text { November } 2007 \\ \text { pp. } 849-854\end{array}\end{array}$

\title{
Probing space-time structure of new physics with polarized beams at the international linear collider
}

\author{
B ANANTHANARAYAN \\ Centre for High Energy Physics, Indian Institute of Science, Bangalore 560 012, India \\ E-mail: anant@cts.iisc.ernet.in
}

\begin{abstract}
At the international linear collider large beam polarization of both the electron and positron beams will enhance the signature of physics due to interactions that are beyond the standard model. Here we review our recently obtained results on a general model-independent method of determining for an arbitary one-particle inclusive state the space-time structure of such new physics through the beam polarization dependence and angular distribution of the final state particle.
\end{abstract}

Keywords. $e^{+} e^{-}$collisions; polarization; beyond the standard model interactions.

PACS Nos 13.88.+e; 13.66.-a; 12.60.-i

\section{Introduction}

At the international linear collider, the possibility of considerable beam polarization has led to a series of investigations on using this as a diagnostic aid for new physics arising due to beyond the standard model interactions (BSM) (for a recent review, see ref. [1]). We recently considered the possibility of observing CP-violating asymmetries in $t \bar{t}$ production with transversely polarized beam [2]. It was shown that only interactions that transform under the Lorentz transformations as pseudoscalar $(\mathrm{P})$, scalar $(\mathrm{S})$ and tensor $(\mathrm{T})$ interactions could contribute. The fact that axial-vector $(\mathrm{A})$ and vector $(\mathrm{V})$ interactions will not contribute to $\mathrm{CP}$-violating asymmetries with transverse beam polarization could have partly been deduced from some general results available in the literature for a general single-particle inclusive process, albeit for the case that the 'new physics' amplitudes interfere with the QED part of the standard model (SM) amplitudes [3].

These general results do not directly apply to other processes of interest, e.g., $Z \gamma$ production, where the SM production goes via $t$ - and $u$-channel amplitudes, in contrast to the case of $t \bar{t}$ production where the SM production goes via $s$-channel amplitudes. Therefore, the general results available in the literature do not apply to the latter process and a case by case study has had to be performed [4-6]. 


\section{B Ananthanarayan}

Indeed, as mentioned earlier, general results involving both the QED as well as the neutral current amplitudes for single-particle inclusive process would be of general interest, as well as the extension to $t$ - and $u$-channel processes. Here we review our recently obtained results on the subject [7]. It may also be noted that our results are sufficiently general to permit a discussion of features of, e.g., chargino and neutralino production in the minimal supersymmetric standard model (MSSM).

We note here that we do not give an extended bibliography on the subject and instead refer to the same in ref. [7].

\section{Correlations and their features}

The process of interest to us here is the one-particle inclusive process

$$
e^{-}\left(p_{-}\right)+e^{+}\left(p_{+}\right) \rightarrow H(p)+X
$$

where $H$ is a final-state particle, whose momentum $p$ is measured, but not the spin, and $X$ is an inclusive state. The process is assumed to occur through an $s$-channel exchange of a $\gamma$ and a $Z$ in the SM, and through a new current whose coupling to $e^{+} e^{-}$can be of the type $\mathrm{V}, \mathrm{A}$, or $\mathrm{S}, \mathrm{P}$, or $\mathrm{T}$. We calculate the relevant factor in the interference between the standard model currents with the BSM currents as

$$
\operatorname{Tr}\left[\left(1-\gamma_{5} h_{+}+\gamma_{5} \phi_{+}\right) \not p_{+} \gamma_{\mu}\left(g_{\mathrm{V}}^{e}-g_{\mathrm{A}}^{e} \gamma_{5}\right)\left(1+\gamma_{5} h_{-}+\gamma_{5} \phi_{-}\right) \not p_{-} \Gamma_{i}\right] H^{i \mu} .
$$

Here $g_{\mathrm{V}}^{e}, g_{\mathrm{A}}^{e}$ are the vector and axial-vector couplings of the photon or $Z$ to the electron current, and $\Gamma_{i}$ is the corresponding coupling to the new physics current, $p_{ \pm}$are the four-momenta of $e^{ \pm}, h_{ \pm}$are the helicities (in units of $\frac{1}{2}$ ) of $e^{ \pm}$, and $s_{ \pm}$ are respectively their transverse polarizations. For details on the notation which are spelt out in great detail, see ref. [7]. We should of course add the contributions coming from photon exchange and $Z$ exchange, with the appropriate propagator factors. However, we give here the results for $Z$ exchange, from which the case of photon can be deduced as a special case. The tensor $H^{i \mu}$ stands for the interference between the couplings of the final state to the SM current and the new physics current, summed over final-state polarizations, and over the phase space of the unobserved particles $X$. It is only a function of the momenta $q=p_{-}+p_{+}$and $p$. The implied summation over $i$ corresponds to a sum over the forms $\mathrm{V}, \mathrm{A}, \mathrm{S}, \mathrm{P}, \mathrm{T}$, together with any Lorentz indices that these may entail.

We now determine the forms of the matrices $\Gamma_{i}$ and the tensors $H^{i \mu}$ in the various cases, using only Lorentz covariance properties. We set the electron mass to zero. Consider now the three cases:

1. Scalar and pseudoscalar case: In this case, there is no free Lorentz index for the leptonic coupling. Consequently, we can write it as

$$
\Gamma=g_{\mathrm{S}}+i g_{\mathrm{P}} \gamma_{5} \text {. }
$$

The tensor $H^{i \mu}$ for this case has only one index, viz., $\mu$. Hence the most general form for $H$ is

$$
H_{\mu}^{\mathrm{S}}=F\left(q^{2}, p \cdot q\right) p_{\mu},
$$


where $F$ is a function of the Lorentz-invariant quantities $q^{2}$ and $p \cdot q$.

2. Vector and axial-vector case: The leptonic coupling for this case can be written as

$$
\Gamma_{\mu}=\gamma_{\mu}\left(g_{\mathrm{V}}-g_{\mathrm{A}} \gamma_{5}\right) .
$$

The tensor $H$ for this case has two indices, and can be written as

$$
H_{\mu \nu}^{\mathrm{V}}=-g_{\mu \nu} W_{1}\left(q^{2}, p \cdot q\right)+p_{\mu} p_{\nu} W_{2}\left(q^{2}, p \cdot q\right)+\epsilon_{\mu \nu \alpha \beta} q^{\alpha} p^{\beta} W_{3}\left(q^{2}, p \cdot q\right),
$$

where now there are three invariant functions, $W_{1}, W_{2}, W_{3}$.

3. Tensor case: In the tensor case, the leptonic coupling is

$$
\Gamma_{\mu \nu}=g_{\mathrm{T}} \sigma_{\mu \nu}
$$

The tensor $H$ for this case can be written in terms of the four invariant functions $F_{1}, F_{2}, P F_{1}, P F_{2}$ as

$$
\begin{aligned}
H_{\mu \rho \tau}^{\mathrm{T}}= & \left(q_{\rho} p_{\tau}-q_{\tau} p_{\rho}\right) p_{\mu} F_{1}\left(q^{2}, p \cdot q\right)+\left(g_{\rho \mu} p_{\tau}-g_{\tau \mu} p_{\rho}\right) F_{2}\left(q^{2}, p \cdot q\right) \\
& +\epsilon_{\rho \tau \alpha \beta} p^{\alpha} q^{\beta} p_{\mu} P F_{1}\left(q^{2}, p \cdot q\right)+\epsilon_{\rho \tau \mu \alpha} p^{\alpha} P F_{2}\left(q^{2}, p \cdot q\right) .
\end{aligned}
$$

Evaluating the trace in each case, we present the results in tables $1-3$, with $\vec{K} \equiv$ $\left(\vec{p}_{-}-\vec{p}_{+}\right) / 2=E \hat{z}$, where $\hat{z}$ is a unit vector in the $z$-direction, $E$ is the beam energy, and $\vec{s}_{ \pm}$lie in the $x-y$ plane for $g_{\mathrm{A}}^{e}$ alone. The tables corresponding to $g_{\mathrm{V}}^{e}$ alone are not given, and was the case considered in ref. [3] for the interference of QED amplitudes with physics due to the then undetermined amplitude of the neutral current due to $Z$.

In tables 1-3, are also given the charge conjugation $C$ and parity $P$ properties of the various correlations, under the assumption that the final-state particle observed is self-conjugate, viz., $H=\bar{H}$. If it is not self-conjugate, then the $C$ factor given in the tables would apply to the sum of the cross-sections for production of $H$ and $\bar{H}$. The difference of these cross-sections would take a $C$ factor of the opposite sign. The counting of the number of independent correlations for the vector and axial-vector cases turns out to be subtle, and is described at length in ref. [7].

We might like to use the behaviour of the differential cross-section to construct asymmetries which can test symmetry properties like CP. Tables 1-3 may be employed to make some predictions for what to expect. It is possible to make general deductions in the special case when the final state is a two-particle state. Within that, we consider two possibilities:

Table 1. List of S, P correlations for $g_{\mathrm{A}}^{e}$.

\begin{tabular}{llcr}
\hline Term & Correlation & $P$ & $C$ \\
\hline $\operatorname{Im}\left(g_{\mathrm{P}} F\right)$ & $2 E^{2}\left(h_{+} \vec{s}_{-}+h_{-} \vec{s}_{+}\right) \cdot \vec{p}$ & + & + \\
$\operatorname{Im}\left(g_{\mathrm{S}} F\right)$ & $2 E\left[\vec{K} \cdot\left(h_{+} \vec{s}_{-}-h_{-} \vec{s}_{+}\right) \times \vec{p}\right]$ & - & + \\
$\operatorname{Re}\left(g_{\mathrm{S}} F\right)$ & $2 E^{2} \vec{p} \cdot\left(\vec{s}_{+}+\vec{s}_{-}\right)$ & - & + \\
$\operatorname{Re}\left(g_{\mathrm{P}} F\right)$ & $2 E\left[\vec{K} \cdot\left(\vec{s}_{+}-\vec{s}_{-}\right) \times \vec{p}\right]$ & + & + \\
\hline
\end{tabular}




\section{$B$ Ananthanarayan}

Table 2. List of V, A correlations for $g_{\mathrm{A}}^{e}$.

\begin{tabular}{llrr}
\hline Term & Correlation & $P$ & $C$ \\
\hline $\operatorname{Re}\left(g_{\mathrm{V}} W_{1}\right)$ & $4 E^{2}\left(h_{+}-h_{-}\right)$ & - & - \\
$\operatorname{Re}\left(g_{\mathrm{A}} W_{1}\right)$ & $-4 E^{2}\left(h_{+} h_{-}-1\right)$ & + & + \\
$\operatorname{Re}\left(g_{\mathrm{V}} W_{2}\right)$ & $2\left(\vec{K} \cdot \vec{K} \vec{p} \cdot \vec{p}-(\vec{p} \cdot \vec{K})^{2}\right)\left(h_{+}-h_{-}\right)$ & - & - \\
$\operatorname{Re}\left(g_{\mathrm{A}} W_{2}\right)$ & $-2\left[-2 E^{2} \vec{p} \cdot \vec{s}-\vec{p} \cdot \vec{s}_{+}+\left(\vec{K} \cdot \vec{K} \vec{p} \cdot \vec{p}-(\vec{p} \cdot \vec{K})^{2}\right)\right.$ & & \\
& $\left.\left(h_{+} h_{-}-1+\vec{s}_{+} \cdot \vec{s}-\right)\right]$ & + & + \\
$\operatorname{Im}\left(g_{\mathrm{V}} W_{3}\right)$ & $-8 E^{2}(\vec{p} \cdot \vec{K})\left(h_{+} h_{-}-1\right)$ & + & - \\
$\operatorname{Im}\left(g_{\mathrm{A}} W_{3}\right)$ & $8 E^{2}(\vec{p} \cdot \vec{K})\left(h_{+}-h_{-}\right)$ & - & + \\
$\operatorname{Im}\left(g_{\mathrm{V}} W_{2}\right)$ & $-2 E\left(\vec{p} \cdot \vec{s}+\left[\vec{K} \cdot \vec{s}_{-} \times \vec{p}\right]+\vec{p} \cdot \vec{s}_{-}\left[\vec{K} \cdot \vec{s}_{+} \times \vec{p}\right]\right)$ & - & - \\
\hline
\end{tabular}

Table 3. List of $\mathrm{T}$ correlations for $g_{\mathrm{A}}^{e}$.

\begin{tabular}{llll}
\hline Term & Correlation & $P$ & $C$ \\
\hline $\operatorname{Im}\left(g_{\mathrm{T}} F_{1}\right)$ & $-8 E^{2} \vec{p} \cdot \vec{K}\left[\vec{p} \cdot\left(\vec{s}_{+}+\vec{s}_{-}\right)\right]$ & - & - \\
$\operatorname{Im}\left(g_{\mathrm{T}} F_{2}\right)$ & $-4 E^{2} \vec{p} \cdot\left(\vec{s}+\vec{s}_{-}\right)$ & - & - \\
$\operatorname{Im}\left(g_{\mathrm{T}} P F_{1}\right)$ & $-8 E \vec{p} \cdot \vec{K}\left[\vec{K} \cdot\left(\vec{s}_{+}-\vec{s}_{-}\right) \times \vec{p}\right]$ & + & - \\
$\operatorname{Im}\left(g_{\mathrm{T}} P F_{2}\right)$ & $4 E\left[\vec{K} \cdot\left(\vec{s}++\vec{s}_{-}\right) \times \vec{p}\right]$ & + & - \\
$\operatorname{Re}\left(g_{\mathrm{T}} F_{1}\right)$ & $8 E \vec{p} \cdot \vec{K}\left[\vec{K} \cdot\left(h_{+} \vec{s}_{-}-h_{-} \vec{s}_{+}\right) \times \vec{p}\right]$ & - & - \\
$\operatorname{Re}\left(g_{\mathrm{T}} F_{2}\right)$ & $-4 E\left[\vec{K} \cdot\left(h_{+} \vec{s}_{-}+h_{-} \vec{s}_{+}\right) \times \vec{p}\right]$ & - & - \\
$\operatorname{Re}\left(g_{\mathrm{T}} P F_{1}\right)$ & $8 E^{2} \vec{p} \cdot \vec{K}\left[\vec{p} \cdot\left(h_{+} \vec{s}_{-}+h_{-} \vec{s}_{+}\right)\right]$ & + & - \\
$\operatorname{Re}\left(g_{\mathrm{T}} P F_{2}\right)$ & $4 E^{2} \vec{p} \cdot\left(h_{+} \vec{s}_{-}-h_{-} \vec{s}_{+}\right)$ & + & - \\
\hline
\end{tabular}

Case 1: $H=\bar{H}$. The simplest case to consider is when $H$ is self-conjugate, i.e., $H=\bar{H}$.

Case 2: $H \neq \bar{H}$. As mentioned earlier, in this case, the $C$ properties in the tables refer to the sum

$$
\Delta \sigma^{+}=\Delta \sigma+\Delta \bar{\sigma}
$$

where $\Delta \sigma$ and $\Delta \bar{\sigma}$ are partial cross-sections corresponding respectively to $H$ and $\bar{H}$ production. The difference of these,

$$
\Delta \sigma^{-}=\Delta \sigma-\Delta \bar{\sigma}
$$

will have the opposite $C$ property.

We have further considered two special cases: when the final state consists of a pair of conjugate particles $H \bar{H}$, and when it consists of two particles $H \bar{H}^{\prime}$, where $H^{\prime} \neq H$.

Case $2 \mathrm{a}: X \equiv \bar{H}$.

Case $2 \mathrm{~b}: X \equiv \bar{H}^{\prime}, H \neq H^{\prime}$.

The specific properties are discussed in great detail in ref. [7]. 


\section{Extensions and applications}

So far we have dealt with a scenario where the SM interactions take place through $s$-channel $\gamma$ and $Z$ exchanges. This is most suitable for production of particles which have no direct coupling to $e^{-}$and $e^{+}$. However, for production of gauge bosons in the SM, which couple directly to $e^{+} e^{-}$, there would be a $t$-channel and/or $u$ channel lepton exchange. In ref. [7] we have provided a detailed discussion on what conclusions one may draw regarding the correlations for this process and the $\mathrm{CP}$ properties thereof, for the particular case of $Z \gamma$ production. The crucial factor in this adaptation is the fact that for $m_{e}=0$, the only contributions which survive correspond to opposite $e^{-}$and $e^{+}$helicities. For, any final-state particles which may be emitted from an electron line with a flip of electron helicity (as for example, a Higgs boson) will have vanishing coupling in the limit of $m_{e}=0$. We are thus left in the most general case with only chirality-conserving combinations of Dirac matrices, sandwiched between electron and positron spinors of opposite helicities. Such a combination of Dirac matrices is a product of odd number of them. For massless spinors, they can always be reduced to a linear combination of $\gamma_{\mu}$ and $\gamma_{\mu} \gamma_{5}$. We are thus back to the case of $\mathrm{V}$ and A couplings in the $s$-channel considered in the foregoing, except that the coefficients $g_{\mathrm{V}}^{e}$ and $g_{\mathrm{A}}^{e}$ would now be replaced by something more complicated. In fact, they could contain tensors constructed out of momenta occurring in the process. It is possible to absorb these tensors into the definition of the $H$ tensor, and the final result would be that we could still use the tables we have obtained so far, with appropriate redefinitions of $g_{\mathrm{V}}^{e}, g_{\mathrm{A}}^{e}$ and the form factors.

One further application presented in ref. [7] is for the S, P and T case, where it is possible to have CP-odd observables, and of the possible ones listed therein, the ones which occur in the special case of lowest-dimensional observables are $\operatorname{Re}\left(g_{\mathrm{P}} F\right)$ and $\operatorname{Re}\left(g_{\mathrm{T}} F_{2}\right)$, corresponding respectively to the four-Fermi couplings $\operatorname{Im}\left(S_{R R}\right)$ and $\operatorname{Im}\left(T_{R R}\right)$ in the notation of [2]. The special features observed in that work, viz., that the four-Fermi scalar coupling terms occur with only the $g_{\mathrm{A}}^{e}$ coupling at the electron vertex, and that the tensor coupling terms occur with only the $g_{\mathrm{V}}^{e}$ at the electron vertex, are borne out by our general results.

In ref. [7], it was further shown that some features of our treatment can be carried over to an extension of SM, like the MSSM, using as illustrations chargino and neutralino pair productions. We have also considered popular scenarios for BSM physics, resulting from either extra-dimensional models or from non-commutative models.

The work reviewed here is presently being extended to the processes $e^{+} e^{-} \rightarrow$ $h_{1}\left(p_{1}\right) h_{2}\left(p_{2}\right) X$ and to $e^{+} e^{-} \rightarrow h(p, s) X$.

\section{Acknowledgements}

We thank the Council for Scientific and Industrial Research for support during the course of these investigations under scheme number 03(0994)/04/EMR-II, as well as the Department of Science and Technology, Government of India, and K Shivaraj and A Upadhyay for reading the manuscript. 


\section{$B$ Ananthanarayan}

\section{References}

[1] G A Moortgat-Pick et al, arXiv:hep-ph/0507011

[2] B Ananthanarayan and S D Rindani, Phys. Rev. D70, 036005 (2004), arXiv:hep$\mathrm{ph} / 0309260$

[3] G V Dass and G G Ross, Nucl. Phys. B118, 284 (1977)

G V Dass and G G Ross, Phys. Lett. B57, 173 (1975)

[4] B Ananthanarayan, S D Rindani, R K Singh and A Bartl, Phys. Lett. B593, 95 (2004); Erratum, Phys. Lett. B608, 274 (2005), arXiv:hep-ph/0404106

[5] B Ananthanarayan and S D Rindani, Phys. Lett. B606, 107 (2005), arXiv:hep$\mathrm{ph} / 0410084$

[6] B Ananthanarayan and S D Rindani, J. High Energy Phys. 0510, 077 (2005), arXiv:hep-ph/0507037

[7] B Ananthanarayan and S D Rindani, Euro. Phys. J. C46, 705 (2006), arXiv:hep$\mathrm{ph} / 0601199$ 